\title{
Ex vivo lung perfusion
}

\section{Tatsuaki Watanabe, Marcelo Cypel, Shaf Keshavjee}

Division of Thoracic Surgery, Toronto Lung Transplant Program, Toronto General Hospital, University Health Network, University of Toronto, Toronto, ON, Canada

Contributions: (I) Conception and design: All authors; (II) Administrative support: All authors; (III) Provision of study materials or patients: All authors; (IV) Collection and assembly of data: All authors; (V) Data analysis and interpretation: All authors; (VI) Manuscript writing: All authors; (VII) Final approval of manuscript: All authors.

Correspondence to: Shaf Keshavjee, MD. Toronto General Hospital, 200 Elizabeth St, 9N-946, Toronto, ON M5G 2C4, Canada.

Email: shaf.keshavjee@uhn.ca.

\begin{abstract}
Lung transplantation is a life-saving treatment for patients with end stage lung disease. The imbalance between lung graft supply and recipients has been a serious issue and barrier to successful lung transplantation. Ex vivo lung perfusion is a strategy wherein lungs are perfused and ventilated outside of the body. This technology has emerged as a safe preservation method that also enables the reassessment and reconditioning of marginal lung grafts. Ex vivo lung perfusion has successfully expanded the donor pool and led to greater lung transplant activity worldwide. Furthermore, ex vivo lung perfusion can be used as a platform for advanced diagnostics that enable specific targeted or personalized treatments that can be developed along a bench to bedside pathway leading to safe ex vivo intervention. Recent findings have shown that ex vivo lung perfusion could significantly and safely extend the preservation period, which enables transplant programs further optimization of the logistics around transplantation surgeries, and create a new paradigm whereby donor lungs are assessed at a centralized ex vivo lung perfusion center prior to delivery to a transplant clinic in need. The introduction of ex vivo lung perfusion to clinical lung transplantation has been a major step in the evolution and practice of lung transplantation.
\end{abstract}

Keywords: Ex vivo lung perfusion; extended criteria donor; donation after cardiocirculatory death donor

Submitted Mar 15, 2021. Accepted for publication Jun 21, 2021.

doi: $10.21037 /$ jtd-2021-23

View this article at: https://dx.doi.org/10.21037/jtd-2021-23

\section{Introduction}

Lung transplantation is a life-saving treatment for patients with various end stage lung diseases. However, a shortage of suitable lung grafts has been a major limiting factor, leading to significant mortality on waiting lists; the death rate is as high as $15-20 \%(1,2)$. Major causes of the insufficient graft supply include the low number of neurologically determined death donors (NDD) and the low rate of acceptable donor lung grafts $(18.6-30 \%)(3,4)$. Several strategies to expand the utilization of acceptable lung grafts have been applied, including single lung transplantation, cadaveric and living donor lobar lung transplantations, the use of extended criteria donors (ECD), and donation after cardiocirculatory death donors (DCD). Ex vivo lung perfusion (EVLP) has emerged as a new solution to increase transplantable lung grafts. EVLP is a strategy wherein donor lungs are perfused and ventilated outside of the body. EVLP has successfully expanded lung graft pools by reassessing lung grafts from ECD and DCD with comparable short- and long-term outcomes $(5,6)$.

Lung grafts are exposed to various injuries during the process of brain death and cardiocirculatory death, such as pneumonia, ventilator associated lung injury, and neurogenic and hydrostatic pulmonary edema. Moreover, lung grafts from DCD can experience additional injuries, including aspiration, warm ischemia, hypoxemia, and hypotension (shock lung). Primary graft dysfunction (PGD), which derives from ischemia-reperfusion injury plus other underlying graft injuries, impairs graft quality that, in turn, 
has been shown to affect short- and long-term morbidity and mortality (7-9). Many lung transplant programs are reluctant to use ECD and DCD lung grafts due to the risk of PGD, and this compounds the problem of insufficient lung graft supply.

EVLP has been developed as an advanced strategy to address the shortage of transplantable lung grafts. EVLP enables the precise reassessment of lungs from ECD and DCD donors, enhances the management of logistical issues by extending preservation time safely $(10,11)$, and provides a platform for advanced molecular diagnosis (12), specific targeted treatments (13-15) and injured lung graft repair (16). This chapter reviews the rationale for EVLP, as well as a discussion of different protocols, perspectives as an advanced diagnostic and organ repair platform, and its use as a logistical enhancement tool.

\section{Rationale behind the method of EVLP}

The current clinical practice for donor lung preservation is cold static preservation $(17,18)$. The induced hypothermia protects lung grafts by reducing cell metabolism, oxygen requirement and nutrient consumption. On the other hand, cold static preservation presents several limitations, including an inability for physiological assessment and the identification of hidden lung injuries which, along with reperfusion injury, can manifest as PGD.

Normothermic EVLP overcomes these issues by enabling cellular metabolic function via a supply of oxygen and nutrients. EVLP is a form of lung preservation with real-time physiological assessment that can also support the recovery of lung grafts.

The development of contemporary EVLP can be divided into three steps: (I) concept of ex vivo organ perfusion; (II) short periods of EVLP with optimized colloid pressure solution; and (III) long periods of EVLP with lung protective ventilation and perfusion.

Leonardo da Vinci first described isolated organ perfusion in his sketches from the fifteenth century. Alexis Carrel and Charles Lindbergh proposed the idea of ex vivo organ perfusion in 1935 (19). Isolated EVLP was first described in 1970 by Jirsch et al., detailing the preservation and evaluation of lungs in cases of distant procurement (20). Unfortunately, those predecessors' attempts at EVLP failed due to the inability to maintain the alveolar-capillary barrier, leading to the development of edema and increased pulmonary vascular resistance-related injuries.
Thirty years later, the contemporary EVLP concept was introduced by Steen and colleagues (21). They overcame issues regarding the alveolar-capillary barrier via the development of STEEN Solution ${ }^{\mathrm{TM}}$ (XVIVO Perfusion, Goteborg, Sweden), a buffered extracellular solution with optimized colloid pressure, which serves as the lung perfusate. This preservation solution maintains fluid within the intravascular space and provides nutrients necessary for pulmonary homeostasis during perfusion (21-23). In 2006, Steen used a short period of EVLP to assess lung grafts prior to successful transplantation (24). However, because of circuit-induced lung injuries, the EVLP technique did not result in a stable preservation period longer than 60 minutes (25).

Thereafter, the Toronto group modified this EVLP technology by using an optimal lung protective strategy with low tidal volume ventilation and a low flow rate combined with a centrifuge pump. The Toronto EVLP method achieved an extended period of stable perfusion of more than 12 hours (26). During Toronto EVLP, lung grafts can be re-evaluated and reconditioned by decreasing extravascular water, reducing harmful and toxic waste products, and recruiting atelectatic areas. EVLP reconditioning results in better ventilation/perfusion matching, and improved microcirculation (26,27). In 2011, the Toronto group reported the successful use of lung allografts from ECD after using their EVLP protocol (6). Subsequently, several promising clinical trials have been performed worldwide $(6,11,28,29)$. Currently, EVLP has become a part of clinical practice in North America, Europe, and Australia (30-33).

\section{EVLP protocols}

EVLP is a concept that includes the perfusion of donor lungs outside of the body using a device that features cellular or acellular solutions, ventilation and normothermia. Three EVLP protocols are currently used worldwide: Toronto, Lund, and Organ Care System ${ }^{\mathrm{TM}}\left(\mathrm{OCS}^{\mathrm{TM}}\right)$ (Transmedics, Andover, MA, USA). The protocols share some similarities; however, they differ in several aspects, such as components of the perfusate, target flow, target pulmonary artery (PA) pressure, open or closed left atrium (LA), and ventilator settings. In the Toronto and Lund protocols, the lung grafts are preserved in a conventional cold static method and are connected to an EVLP system at the recipient hospital or a centralized facility. On the other hand, the OCS Lung is a portable EVLP system, and the lungs are connected to this system at the donor hospital. Currently, most transplant 


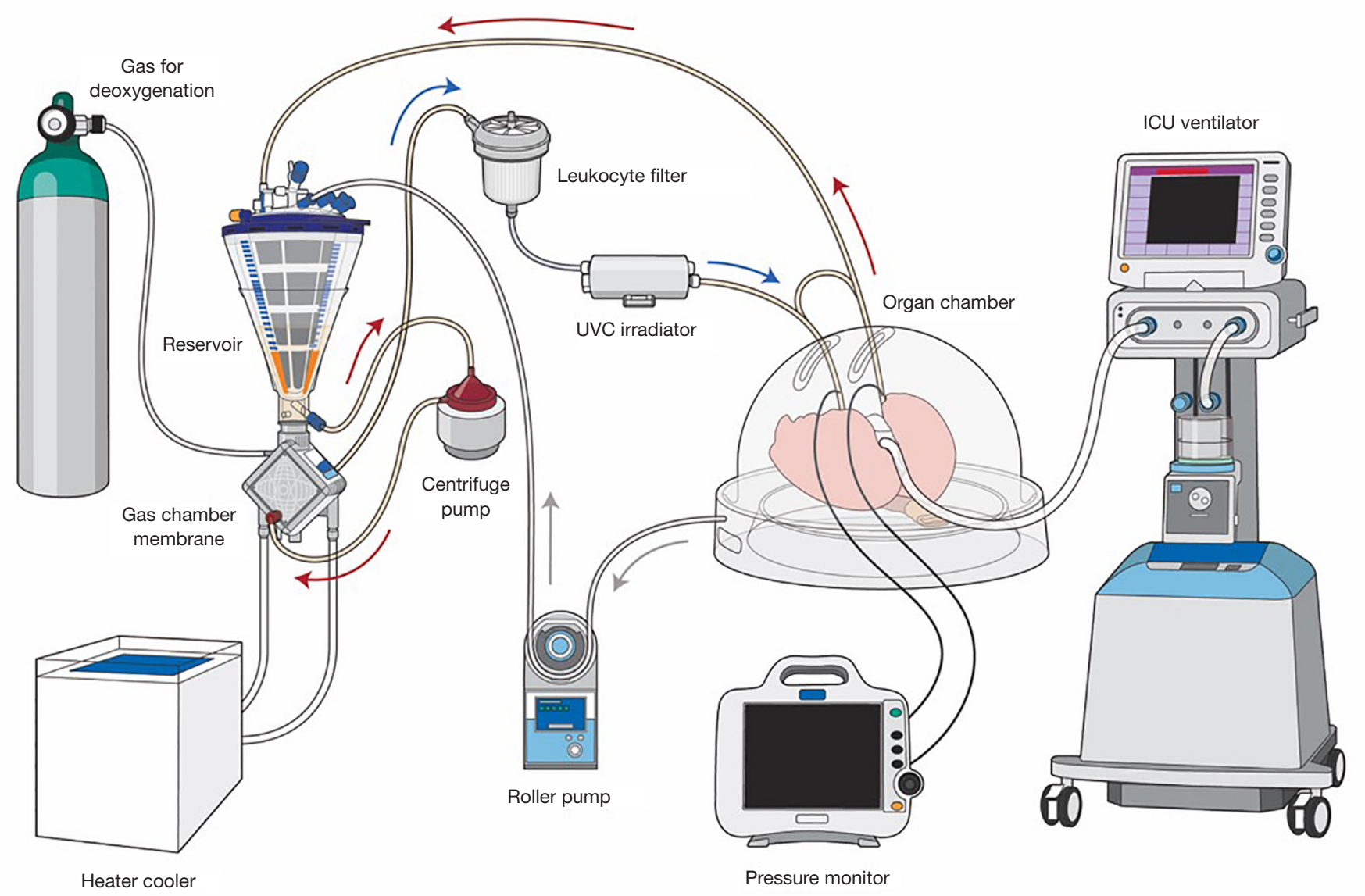

Figure 1 The schema of Toronto EVLP. Gas for deoxygenation consists of $86 \% \mathrm{~N}_{2}, 8 \% \mathrm{CO}_{2}, 6 \% \mathrm{O}_{2}$. *, bridge between inflow and outflow. The bridge is closed during perfusion. The bridge can be used for de-air from inflow line. Arrows indicate the direction of perfusate flow. Arrow colors indicate oxygenation of the perfusate; red indicates oxygenated perfusate and blue indicates de-oxygenated perfusate. UVC irradiator is optional. EVLP, ex vivo lung perfusion; UVC, ultraviolet C.

programs employ the Toronto or OCS Lung protocol, and some employ one of these techniques with additional modifications $(34,35)$.

\section{Toronto EVLP equipment}

Toronto EVLP is performed in an operating room with a team comprised of a surgeon experienced with EVLP, surgical assistants, a perfusionist and a circulating operating room nurse. The Toronto EVLP circuit includes a centrifugal pump, heater/cooler, tubes, reservoir, membrane oxygenator, leukocyte filter, flow censor, pressure transducers, ventilator, bronchoscope system with a monitor, mixture of gases $\left(86 \% \mathrm{~N}_{2}, 8 \% \mathrm{CO}_{2}, 6 \% \mathrm{O}_{2}\right)$ with a cylinder, specific cannulas, and a plastic organ chamber
(26,36) (Figure 1). An ultraviolet C (UVC) irradiator can be applied to inactivate microorganisms in perfusate, such as lungs from hepatitis $\mathrm{C}$ virus ( $\mathrm{HCV}$ ) positive donors (37).

The room for Toronto EVLP should be large enough to house the entire set of components, as well as two surgical tables (i.e., a surgical back table and a table for the organ chamber), additional monitors and a desk (Figure 2A). A nearby gas analyzer is required for perfusate analysis. A secured electronic system is needed to share updated information describing hourly assessments to the transplant team.

Commercially available devices that use the Toronto EVLP protocol include the portable XVIVO Perfusion System $\left(\mathrm{XPS}^{\mathrm{TM}}\right.$ ) (XPS Perfusion, Goteborg, Sweden) and Lung Assist ${ }^{\mathrm{TM}}$ (Organ Assist, Groningen, Netherlands). 


\section{Toronto EVLP technique}

The Toronto EVLP technique is the most widely used and referenced method in clinical lung transplantation and preclinical research to date, primarily due to the ability to perform safe and extended EVLP. The main differences between the Toronto technique and other EVLP methods are: (I) the use of an acellular perfusate, (II) a closed circuit with a positive LA pressure, and (III) low perfusion flow.

After lung graft recovery using a standard cold static preservation technique, the pulmonary bloc is dissected on a back table at the recipient hospital or a centralized center. A specific cuffed LA cannula is trimmed and sewn to the LA using two 4-0 polypropylene running sutures (Figure $2 B$ ). In case of LA injury or a short LA cuff, the cuff can be repaired with a pericardium for cannulation (Figure 2C). If the PA trunk is of sufficient length, the straight cannula is directly inserted and secured with heavy silk ties. In case of a short PA trunk, a specific cuffed PA cannula is trimmed and sewn to the PA using two 5-0 polypropylene running sutures. The trachea is clamped above the first carina level to keep lungs inflated, and the staples are then removed. With its oblique tip removed, a regular endotracheal tube (Figure $2 D$ ) is inserted into the trachea and secured with heavy silk ties (Figure 2D). Then, the endotracheal tube is clamped, and the clamp on the trachea is released. A retrograde flush with $1 \mathrm{~L}$ of Perfadex ${ }^{\circledR}$ solution (XVIVO Perfusion) is performed via the LA cannula at the back table (38).

The lungs are placed in the EVLP dome and the PA cannula is connected to the circuit after de-airing (Figure 2E). Then, the LA cannula is connected to the circuit. The cannulas are attached to the dome without kinking of the PA and pulmonary veins (Figure $2 E$ ). The EVLP circuit is primed with $2 \mathrm{~L}$ of STEEN solution and $500 \mathrm{mg}$ of methylprednisolone, 3,000 IU of unfractionated heparin and $500 \mathrm{mg}$ of imipenem/cilastatin. The lungs are gradually warmed by increasing the perfusate temperature, via setting the heater-cooler to $30{ }^{\circ} \mathrm{C}$ at $10 \mathrm{~min}$ and $37{ }^{\circ} \mathrm{C}$ at $20 \mathrm{~min}$. The perfusate flow rate is set as $10 \%$ of the target flow ( $40 \%$ of cardiac output) at the beginning, and is increased to the maximum flow step by step $(20 \%$ of the target flow at $10 \mathrm{~min}, 30 \%$ at $20 \mathrm{~min}, 50 \%$ at $30 \mathrm{~min}, 80 \%$ at $40 \mathrm{~min}$, and $100 \%$ at $50 \mathrm{~min}$ ). LA pressure is maintained at 3 to $5 \mathrm{mmHg}$ by adjusting the reservoir height. PA pressure is normally $<13 \mathrm{mmHg}$, and determined by perfusate flow, pulmonary vascular resistance, and LA pressure. At 20-30 min after EVLP start, perfusate temperature is gradually increased. When the perfusate temperature reaches $32{ }^{\circ} \mathrm{C}$, protective ventilation $\left(\mathrm{FiO}_{2}\right.$ of $21 \%$, tidal volume of $7 \mathrm{~mL} / \mathrm{kg}$, respiratory rate of 7 breaths $/ \mathrm{min}$, and a positive end-expiratory pressure ( $\mathrm{PEEP}$ ) of $5 \mathrm{cmH}_{2} \mathrm{O}$ ) is targeted, followed by a gas mixture flow at $1 \mathrm{~L} / \mathrm{min}$. The post-membrane partial pressure of $\mathrm{CO}_{2}$ is adjusted between 35 and $40 \mathrm{mmHg}$ by titrating the sweep gas flow. A lung recruitment maneuver (up to $25 \mathrm{cmH}_{2} \mathrm{O}$ of peak airway pressure or $15 \mathrm{~mL} / \mathrm{kg}, 20 \mathrm{sec}, 2$ times) is performed at $60 \mathrm{~min}$ of perfusion and then between hourly assessments. STEEN solution is partially exchanged hourly: $500 \mathrm{~mL}$ at the first hour and $250 \mathrm{~mL}$ at every hour thereafter. Hourly lung function evaluation is conducted after 5 minutes of ventilation on $100 \% \mathrm{FiO}_{2}$, tidal volume of $10 \mathrm{~mL} / \mathrm{kg}$, PEEP of $5 \mathrm{cmH}_{2} \mathrm{O}$, and respiratory rate of $10 / \mathrm{min}$. Hourly lung evaluation is performed by measuring the ratio of $\mathrm{PaO}_{2}$ and fraction of inspired oxygen $\left(\mathrm{PaO}_{2} / \mathrm{FiO}_{2}\right.$ ratio $)$ in LA effluent and PA influent, along with PA pressure, dynamic and static compliances, peak airway pressures, and hourly loss of STEEN solution. Lung radiography and flexible bronchoscopy are routinely performed at 1 hour and 3 hours of EVLP (38). When localized lung injuries are suspected, a differential measurement of the $\mathrm{PaO}_{2} / \mathrm{FiO}_{2}$ ratio is performed by sampling perfusate from each pulmonary vein.

At the end of EVLP, the lung block is cooled down to $10{ }^{\circ} \mathrm{C}$ in the circuit over a 10 -minute period. Thereafter, perfusion and ventilation are stopped $\left(\mathrm{FiO}_{2}\right.$ is increased to $50 \%$ for lung storage), and the tracheal tube is clamped to keep the lungs inflated at $7 \mathrm{~mL} / \mathrm{kg}$ and PEEP of $5 \mathrm{cmH}_{2} \mathrm{O}$. The trachea is stapled, and an antegrade flush is performed with $1.5 \mathrm{~L}$ of cold Perfadex solution via the PA cannula. The PA and LA cannula are then removed, and the lungs are stored with a conventional cold static preservation method until implantation.

\section{Toronto EVLP donor and acceptance criteria}

Most transplant groups use the Toronto EVLP technique to reassess and recondition ECD and DCD lung grafts. The criteria for using Toronto EVLP are summarized in Table 1. The Vienna group demonstrated that Toronto EVLP-assessed lungs from standard criteria donors safely extended preservation time with equivalent outcomes to the standard cold preservation (11). The basic criteria for lung graft acceptance for lung transplantation after Toronto EVLP are shown in Table 2. The decision to transplant is made by using a set of acceptance criteria, including delta 

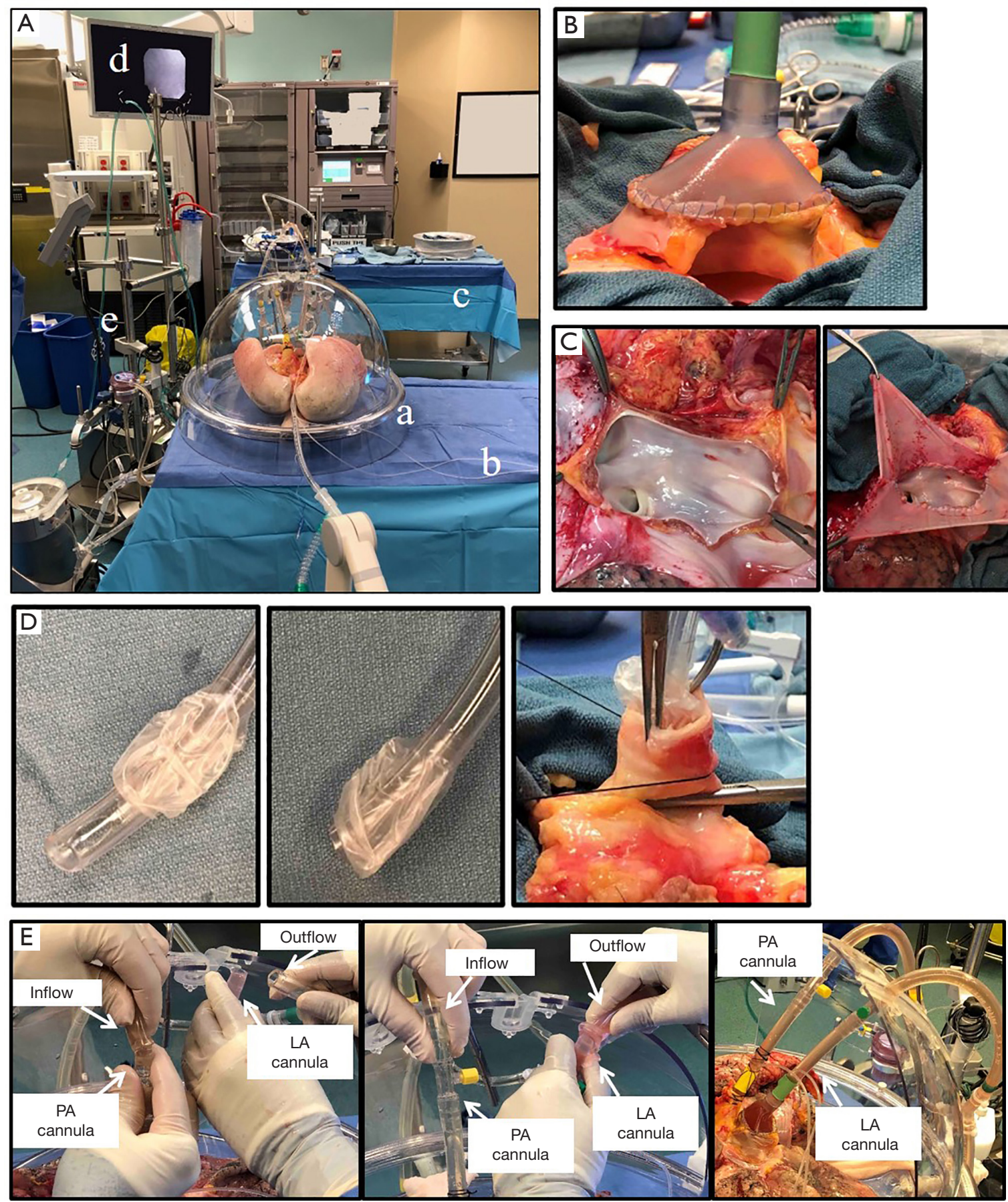

Figure 2 Toronto EVLP technique. (A) Toronto EVLP system. a: organ chamber; b: table for organ chamber; c: back table; d: monitor for bronchoscope; e: EVLP circuit. (B) LA and LA cannula connection. LA and LA cannula are sewn with 4-0 polypropylene sutures. (C) In the case of a short LA. Left: short LA. Right: LA is fixed with pericardium and 4-0 polypropylene sutures before cannulation. (D) Left and middle: the tip of endotracheal tube was removed. Right: the trachea was closed with a clamp and the endotracheal tube is fixed to the trachea with heavy silk ties. (E) Connection of PA and LA cannulas to the circuit. Left: connecting Inflow tube to PA cannula after de-air PA cannula and pulmonary vasculature. LA cannula was open at this time. Middle: connecting outflow tube and LA cannula. Clamp on outflow cannula was removed immediately after connection. Right: cannulas and circuit were connected. PA and LA cannulas were fixed to the chamber without kinking of PA and pulmonary veins. EVLP, ex vivo lung perfusion; LA, left atrium; PA, pulmonary artery. 
Table 1 Indication for EVLP

Best $\mathrm{PaO}_{2} / \mathrm{FiO}_{2}$ lower than $300 \mathrm{mmHg}$

Presence of pulmonary edema: on chest X-ray or in the clinical assessment

Poor lung/lobe compliance

High risk history

Lung from marginal DCD

DCD, donor. The table is modified from Ref. (36). EVLP, ex vivo lung perfusion; WLST, withdrawal of life support therapy.

Table 2 Acceptance and exclusion criteria after 4-6 h of ex vivo lung perfusion

Acceptance criteria
Delta $\mathrm{pO}_{2} \geq 350 \mathrm{mmHg}$
Stable or improving pulmonary artery pressure
Stable or improving airway pressures
Stable or improving pulmonary compliance
Acceptable STEEN solution loss
Exclusion criteria
Delta pO $\mathrm{O}_{2}<350$ mmHg
$>15 \%$ deterioration on pulmonary artery pressure
$>15 \%$ deterioration on airway pressure
$>15 \%$ deterioration on pulmonary compliance

The table is modified from Ref. (38). Delta $\mathrm{pO}_{2}$, difference of $\mathrm{PaO}_{2}$ between samples from left atrium and pulmonary artery under $\mathrm{FiO}_{2}$ of 1.0 .

$\mathrm{pO}_{2}$, lung compliance, $\mathrm{X}$-ray findings, bronchoscopic findings, and physical assessment (39).

\section{Outcome of Toronto EVLP}

Even with prolonged graft preservation, EVLP provides equivalent short-term outcomes (6), functional outcomes (40), and long-term outcomes (5). Moreover, the Toronto group reported successful utilization of lungs from uncontrolled DCD (non-perfused organ donor) with up to 3 hours of warm ischemia followed by EVLP assessment (41). Clinical use of EVLP has enabled the Toronto program to expand their annual lung transplantation activity by $70 \%$. Moreover, the experience has enhanced confidence in the utilization of DCD lungs and high-risk NDD lungs, with an average of $70 \%$ utilization of donor lungs treated with EVLP. Outcomes across low- and high-risk DCD, high-risk NDD, and EVLP used for logistical reasons are excellent (42).

\section{OCS lung}

OCS Lung is the first portable EVLP system. OCS Lung is mainly used for standard donor lungs, and addresses issues regarding cold ischemic storage by maintaining the organ in a normothermic and physiologically functioning state throughout transportation. OCS Lung differs from the Toronto EVLP technique; it uses a blood-based perfusate with a modified perfusion rate and different pump. The technique also uses an open LA, whereas Toronto EVLP employs a positive left atrial pressure of $3-5 \mathrm{mmHg}$ (Table 1).

In 2012, the Hanover and Madrid groups published a feasibility study using OCS Lung as a primary lung preservation method with standard criteria donors, as an alternative to conventional cold static preservation (32). A combined liver-lung transplantation following stable 675 minutes of EVLP using OCS Lung was reported by the Leuven group in 2014 (43). A prospective, randomized, multicenter trial (INSPIRE trial) was performed from November 2011 to November 2014. Results showed noninferiority of OCS Lung outcomes compared with standard cold preservation (28).

OCS Lung has also been used for lung grafts from ECD and DCD. A prospective, single-arm, multicenter trial evaluating OCS Lung for lungs from ECD and DCD (EXPAND trial) showed $87 \%$ donor lung utilization rate with excellent post-transplant clinical outcomes (29).

\section{EVLP as a platform for specific treatments}

Given that donor lungs can be maintained in a physiologically functioning state prior to transplantation, research studies are now exploring the potential to use EVLP as a platform for advanced therapies to create better lungs for recipients. Lungs are typically perfused for 4 to 6 hours. The Toronto EVLP technique can reliably and reproducibly preserve lungs for 12 hours, which could enable extracorporeal biological treatments that require an extended time period to achieve its desired effect (26). Extension of the EVLP time frame is an ongoing interest in lung transplantation (44) and can permit further advanced diagnostic tests and time-dependent molecular therapies (45). This will improve graft quality and potentially increase the donor lung pool. Recently, stable 
Table 3 EVLP as a platform for specific treatments (13-16,37,48-67)

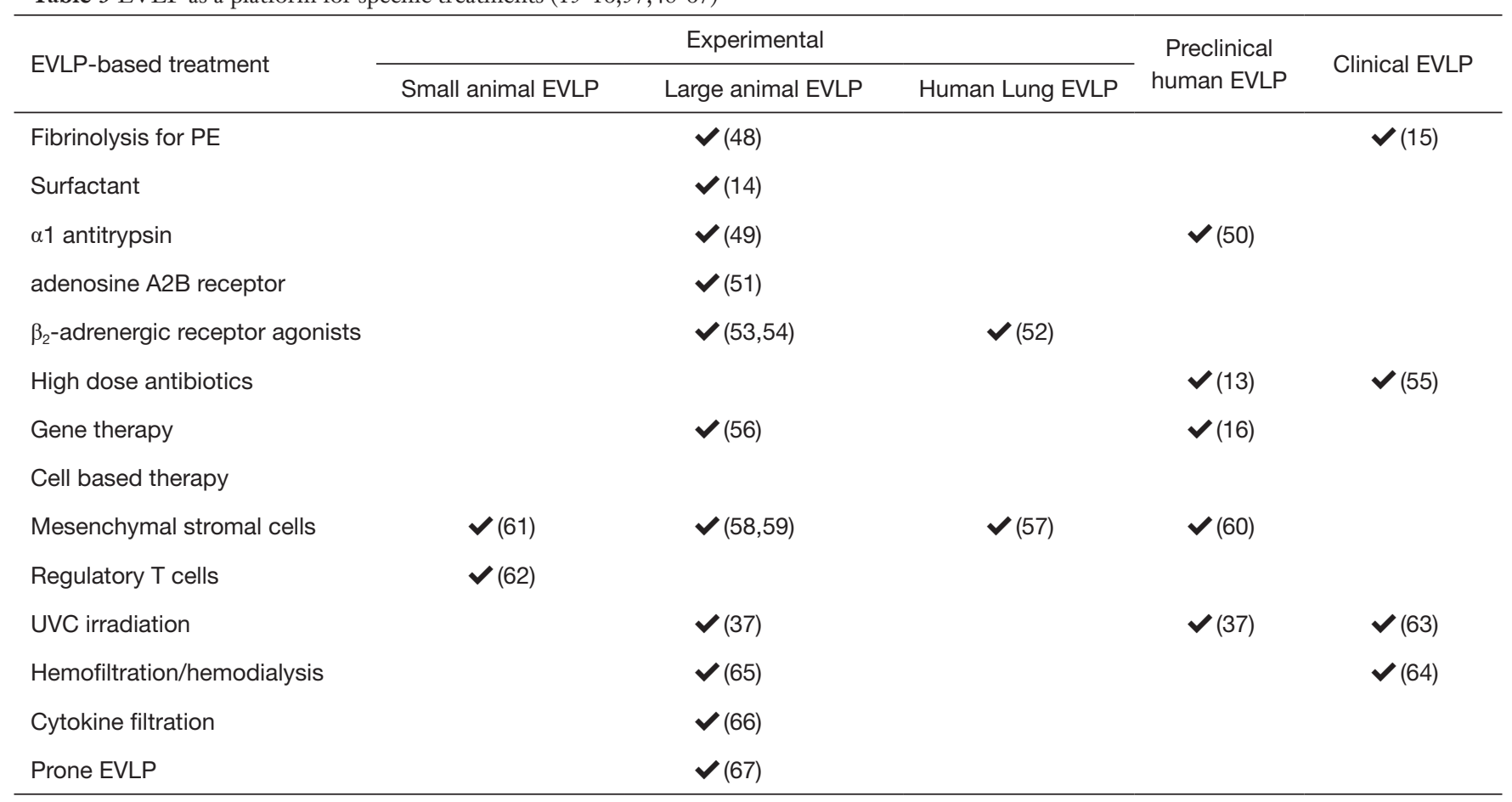

The numbers in the table indicate references. EVLP, ex vivo lung perfusion; PE, pulmonary embolism; UVC, ultraviolet C.

24 hours of EVLP was achieved by supplementing nutritional requirements in both the Toronto EVLP and OCS Lung techniques $(46,47)$.

Several EVLP-based therapeutic approaches have been studied, whereby injuries identified during lung graft assessment on EVLP can inform the application of personalized therapies during the EVLP time window. These therapies include successful administration of drugs, such as antibiotics, fibrinolytic and anti-inflammatory drugs, gene therapy, cell-based therapy, and application of therapeutic devices such as dialysis and an UVC irradiator. Given that the lung graft is isolated on EVLP, each of these strategies can be administered at high concentration via the vasculature or airway without the risk of systemic toxicity. Specific EVLP-based treatments and their current status toward clinical application are described below and summarized in Table 3.

\section{Fibrinolysis for pulmonary embolism}

Lung donors with pulmonary thromboembolism are associated with PGD; removal of the emboli reduces the incidence of PGD (68). Pulmonary embolism can be treated during EVLP. This concept is important for DCD lungs without systemic anticoagulation. In 2007, Inci et al. evaluated the effect of urokinase during a modified EVLP system in a pig DCD model. They demonstrated that $100,000 \mathrm{U}$ of urokinase treatment during EVLP improved pulmonary vascular resistance, oxygenation, and wet-todry ratio (48). They also reported their successful clinical experience of fibrinolysis and administration of 100,000 U of urokinase during EVLP in donor lungs with pulmonary embolism (69). In 2012, Machuca et al. reported the use of alteplase on donor lungs with pulmonary embolism and placed on Toronto EVLP. They administered $20 \mathrm{mg}$ of alteplase into the EVLP circuit and demonstrated successful lung transplantation (15). In 2015, Luc et al. reported the addition of $5 \mathrm{mg}$ of alteplase to OCS lung EVLP for DCD lung grafts with massive pulmonary embolism resulted in successful thrombolysis (70).

\section{Surfactant}

Aspiration is a common event in organ donors (71). Injured lungs as a result of aspiration are not suitable for transplantation because of decreased quality and function, 
and the higher possibility of PGD (72). Several attempts have been made to repair lungs with aspiration injury during EVLP. A team in Zurich used a pig aspiration model to demonstrate the protective effects of lavaging the donor lung with surfactant during EVLP (73), with improved graft function using a second pig aspiration model simulating controlled DCD (74). Nakajima et al. showed that lavage and surfactant administration via the airway during EVLP improved graft function and reduced proinflammatory cytokine expression levels in a pig aspiration model with 10 hours of cold ischemic time (CIT) and 6 hours of EVLP followed by a single left lung transplantation (14).

\section{Pharmacological anti-inflammatory treatment}

$\alpha 1$ antitrypsin (A1AT) is a serine-protease inhibitor that acts as an acute phase reactant during inflammation. Recently, Lin et al. demonstrated that administration of A1AT into EVLP perfusate resulted in improved physiological function with a reduction in the levels of interleukin- $1 \alpha$ (IL-1 $\alpha$ ) and IL-8 (49). Mariscal et al. demonstrated that A1AT administration during EVLP using lungs rejected for clinical transplantation improved oxygenation and reduced pulmonary vascular resistance and perfusate loss. Furthermore, A1AT treatment decreased endothelin-1 level in perfusate and ZO-1 expression on endothelial cells (50).

Adenosine plays a role in purinergic signaling during inflammation. Among its receptors, adenosine A2B receptor activation results in a pro-inflammatory effect in lung ischemia-reperfusion injury. Charles et al. successfully showed the protective effect of an adenosine A2B receptor blocker in a pig model of DCD lungs on EVLP, with 2 hours of warm ischemia followed by lung transplantation (51).

\section{$\beta_{2}$-adrenergic receptor agonists}

$\beta_{2}$-adrenergic receptors are widely distributed in the lungs. Their stimulation leads to the relaxation of bronchial and pulmonary vascular smooth muscle (75). Frank et al. conducted an experiment to measure alveolar fluid clearance using human lungs declined for transplantation. They demonstrated that the basal alveolar fluid clearance was $19 \% / \mathrm{h}$ on EVLP. Intra-airway administration of terbutaline increased alveolar fluid clearance to $43 \% / \mathrm{h}$ per with lower lung weight gain (52). Valenza et al. used a pig EVLP model to demonstrate that infusion of salbutamol in the EVLP circuit decreased mean PA pressure and increased dynamic compliance (53). Recently, the Kyoto group demonstrated that high dose procaterol inhalation during EVLP improved graft function using a canine DCD model (54).

\section{High dose antibiotics}

Lung grafts are vulnerable to bacterial and fungus infection and contamination, which lead to increased morbidity in immunosuppressed recipients (76). EVLP can serve as a platform to apply high dose anti-bacterial and antifungal drug treatment without causing systemic side effects. The Newcastle group demonstrated high dose antibiotics treatment for human lungs on EVLP that were initially considered not suitable for transplantation. They transplanted 6 out of 18 lungs that resulted in no inhospital mortality (55). The Toronto group showed that the use of high dose broad spectrum antibiotics during EVLP significantly reduced bronchoalveolar lavage bacterial counts and endotoxin levels with improved donor lung function (13).

\section{Gene therapy}

The Toronto group has been investigating gene therapy to repair injured human donor lungs-specifically, gene therapy via adenoviral vector encoding human IL-10. Using lungs unsuitable for clinical transplantation, intraairway adenoviral vector encoding human IL-10 treatment during 12 hours of EVLP showed significant improvement in $\mathrm{PaO}_{2} / \mathrm{FiO}_{2}$ ratio and pulmonary vascular resistance, with a favorable shift from pro- to anti-inflammatory cytokine expression and recovered alveolar-blood barriers (16). Machuca et al. performed a pig EVLP experiment with intra-airway adenoviral vector encoding human IL-10 treatment followed by left lung transplantation and 7-day survival. Results showed persistent detection of human IL10 in pig plasma for 7 days and improved graft function at 7 days after lung transplantation. Lymphocytes from the adenoviral vector encoding human IL-10-treated group showed less interferon gamma production at 7 days postlung transplantation (56).

\section{Cell based therapy}

Mesenchymal stromal cells (MSCs) are known to have antiinflammatory and immunomodulatory effects that may ameliorate lung injuries. The use of MSCs for personalized 
therapy on EVLP has been investigated. Matthay's group showed that the instillation of MSCs in human lungs with endotoxin-induced lung injury during EVLP resulted in decreased endothelial permeability, reduced extravascular lung water, and restored alveolar fluid clearance (57).

During EVLP, there are two routes for the administration of MSCs: airway and vasculature. Mordant et al. investigated the optimal route and showed that MSC administration via the vasculature is more effective than the airway (58). Nakajima et al. demonstrated that EVLP MSC treatment via vasculature ameliorated ischemia-reperfusion injury in a pig EVLP model followed by left single lung transplantation (59). Nykanen et al. reported the feasibility of using MSCs genetically engineered to produce antiinflammatory IL-10 and applied into the perfusate in a pig EVLP model with 24 hours of cold static preservation followed by left single lung transplantation with 3 day survival (77). Furthermore, they conducted a preclinical study regarding the application of genetically engineered MSCs during EVLP using human lungs declined for clinical use. Results showed that genetically engineered MSCs were retained in the lung and IL-10 levels in EVLP perfusate were rapidly increased. In an improvement from their previous pre-clinical study, perfusate IL-10 level declined after 4-6 hours of perfusion, particularly in lungs with low perfusate $\mathrm{pH}$ and glucose, and high lactate levels (60).

The Milan group reported that MSC-derived extracellular vesicles decreased pulmonary vascular resistance and wet-dry ratio, and improved molecular phenotype in a rat EVLP model (61).

Miyamoto et al. used EVLP and regulatory $\mathrm{T}$ cells (Tregs) to achieve allograft immune regulation before lung transplantation. They established a rat EVLP model followed by left single lung transplantation with a 3-day survival period. Cryopreserved Tregs applied to the EVLP circuit exerted immunomodulatory effects in vivo and in vitro (62).

\section{Light therapy}

One of the unique aspects of the EVLP system is that external treatment of the circulating perfusate can be used to rehabilitate the ex vivo lung. The Toronto group applied light therapy (i.e., UVC) to perfusate during EVLP in order to observe if there is any effect in minimizing the risk of viremia. Galasso et al. used a pig EVLP transplant model to show the safe reduction of HCV load in HCV-positive donors using UVC light integrated with the EVLP circuit (Figure 1) (37). Furthermore, the group demonstrated the efficacy and safety of lung transplantation from HCVpositive donors to $\mathrm{HCV}$-negative recipients after EVLP combined with $400 \mathrm{mg}$ sofosbuvir and $100 \mathrm{mg}$ velpatasvir treatment after transplantation. There was equivalent 6-month survival in recipients receiving $\mathrm{HCV}$-positive (95\%) vs. HCV-negative donor lungs (94\%). $86 \%$ of patients receiving $\mathrm{HCV}$-positive donor lungs were $\mathrm{HCV}$ free at 6 months after transplantation. In addition, UVC treatment on EVLP showed significant reduction of viral load, and prevented HCV transmission in 2 out of 11 patients (63).

\section{Hemofiltration and cytokine filtration}

During EVLP, osmotic pressure can change, in addition to a reduction in glucose and increases in electrolyte and lactate levels $(49,78)$. In the Toronto EVLP technique, the perfusate is partially replaced every hour. The Gothenburg group applied hemofiltration to maintain osmotic pressure during EVLP (64). Nilsson et al. performed an experiment using a pig lung edema model and found that hemofiltration increased the oncotic pressure of the perfusate and decreased lung weight with a beneficial effect on lung compliance, but did not improve lung oxygenation capacity (65).

The Edmonton group used a pig EVLP negative pressure ventilation model (79) to test if continuous hemodialysis stabilized electrolyte levels during EVLP. They successfully maintained sodium levels in perfusate for over 24 hours of EVLP. However, continuous hemodialysistreated lungs did not show improved graft function or reduced pro-inflammatory cytokine elevation (80).

Cytokines and chemokines are released during lung ischemia-reperfusion injury, activating innate immune cell activity including neutrophil recruitment. This leads to capillary leakage and cell death, which result in subsequent lung allograft damage (81). These cytokines and chemokines accumulate in EVLP perfusate (49). The Okayama group used a pig EVLP model to show that the application of an adsorbent membrane suppressed IL-8 and tumor necrosis factor- $\alpha$ early after EVLP but did not improve lung graft function (82). The Zurich group demonstrated the safety and efficacy of cytokine filtration in a 12-hour pig EVLP model. They showed that cytokine filtration resulted in a significant reduction of multiple cytokines with improved 
EVLP as bench to bedside translation pathway

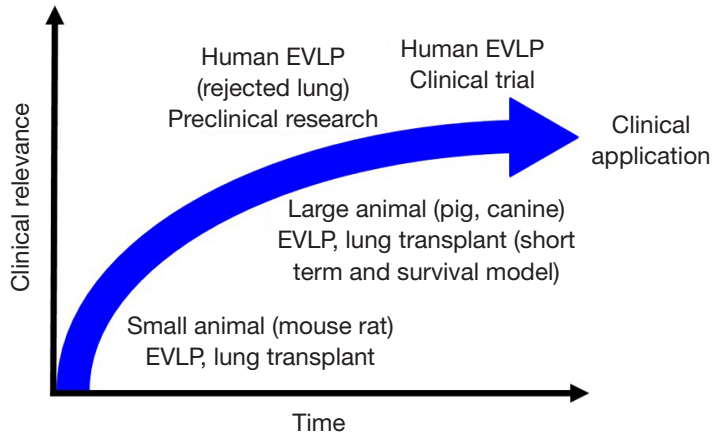

Figure 3 Bench to bedside translation pathway. EVLP can be used to help translate outcomes across physiological conditions in in vitro, small animal, large animal, and human research models. Small animal EVLP can be used to test the effect of drugs in isolated lungs and develop novel therapies with a variety of biochemical assessment tools at a relatively low cost. Large aminal EVLP can be used to test the effects and safety of novel therapies that represents physiologically close conditions to a clinical scenario. EVLP can utilize clinically declined human lungs as a translational step toward clinical application of each investigated therapy. EVLP, ex vivo lung perfusion.

lung graft function (66).

\section{Prone positioning}

The dorsal portion of the lung is more susceptible to atelectasis. Prone positioning has shown beneficial effects for patients with lung injury (83). Prone positioning also improved graft function in a pig DCD EVLP model (84). Niikawa $e t a l$. tested the role of prone positioning of lungs on EVLP and demonstrated better oxygenation and lung compliance, lower weight ratio, and lower IL-1 $\beta$ levels in prone position lungs compared to control lungs in supine position (67).

\section{EVLP as bench to bedside pathway}

Novel pre-clinical treatments to improve lung quality have been extensively studied. However, the translation of these studies to the clinic are challenged by different physiological conditions across in vitro, small animal, large animal, and human models. The use of EVLP as a research tool can help with this bench to bedside knowledge transfer (Figure 3). For example, the Toronto group has investigated the clinical application of A1AT to ameliorate ischemiareperfusion injury after lung transplantation, using EVLP to verify outcomes across pre-clinical models. This includes studying A1AT in cell culture, rat hilum clamp model, and pig lung transplantation with short-term assessment and pig EVLP application (85-87). Together, this has informed the safety and efficacy of A1AT in a pig lung transplant model with 3-day survival (49). As the final step toward clinical application, EVLP using clinically declined human donor lungs served as the final preclinical study environment (50).

For over 20 years, the Toronto group has also been working on IL-10 gene therapy for lung transplantation, using small animal models, preclinical EVLP using rejected human lungs, and pig EVLP followed by lung transplantation with 7 -day survival model $(16,56,88-90)$. This serial progression towards clinical application exemplifies EVLP as an excellent translational research platform.

\section{EVLP as an advanced diagnostic tool}

The assessment of cellular function during cold static preservation is challenging due to the low metabolic state of the lung. In contrast, normothermic EVLP maintains the physiological and metabolic activity of the lung graft. Assessment of graft quality during EVLP is primarily reported via physiological parameters, including ventilation and standard arterial blood gas analyses of perfusate samples. In addition, perfusate analysis, such as glucose consumption and lactate level, enables metabolic state evaluation (78). One of the unique features of EVLP perfusate is that it enables insight into the holistic changes occurring in the lung graft, independent of other organs. Therefore, changes in inflammatory cytokines and cell death markers can be attributed solely to donor lung activity and used as a biomarker to precisely predict post-lung transplantation outcomes.

Machuca et al. reported protein expression levels using clinical EVLP samples divided into three groups: control (good early outcomes); PGD 3; and declined (lungs declined after EVLP). The team concluded that IL-8 and growthregulated oncogene- $\alpha$ best differentiated between control and PGD 3, and stem cell growth factor- $\beta$ differentiated between control and declined lungs (12). They also identified higher endothelin-1 and big endothelin-1 expression in declined lung perfusate compared to control (91). Andreasson et al. reported IL-1 $\beta$ is the most effective marker to differentiate in-hospital survival $v s$. non- 


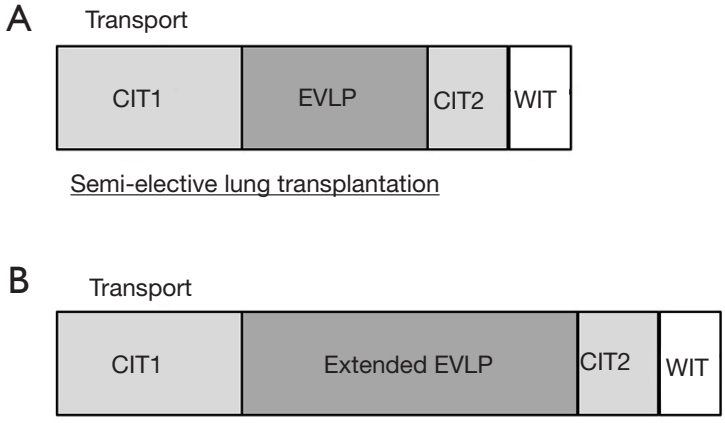

Biological treatment on EVLP

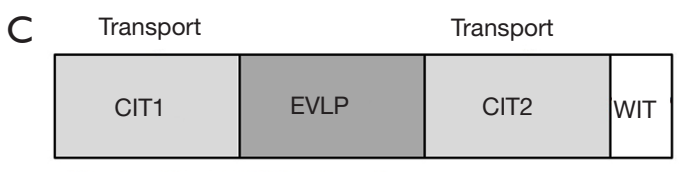

Centralized EVLP system

Figure 4 Schema of emergent clinical applications of EVLP. (A) EVLP as a preservation strategy for semi-elective lung transplantation. (B) EVLP as a therapeutic platform for advanced approaches relevant to organ repair and regeneration. (C) Model for high-volume specialized organ assessment and repair centers. The figure is modified from Ref. (45). EVLP, ex vivo lung perfusion.

survival post-transplant (92).

Hashimoto et al. tested circulating cell death marker expression levels in EVLP perfusate across control (good early outcome) and PGD 3 groups. They demonstrated that M30 (indicating epithelial cell apoptosis) is higher in the PGD 3 group. Also, the increase of high mobility group box-1 from 1 to 4 hours of EVLP was greater in the PGD 3 group (93).

Neutrophil extracellular traps (NETs) are accumulated in experimental and human PGD (94). NETs could serve as additional useful biomarkers of severe PGD and allograft injury at the time of transplant. A recent study showed that NET levels during EVLP correlates with adverse recipient outcomes (95).

Molecular-based diagnosis during EVLP will help to enable the development of rapid diagnostic platforms that will aid in the clinical decision to use EVLP lungs for transplant and predict lung transplant outcomes. Currently, clinical decision-making during EVLP is based on physiological parameters, direct visual assessment, and X-ray findings that, together with subjective clinical judgement, only provide a part of the status of lung injury. Sage et al. developed a cytokine-based score using a novel rapid testing device to assist lung transplant team decision-making. They conducted a retrospective study using biobanked clinical EVLP perfusate samples to develop biological mediators that predict: (I) if a lung would be acceptable for transplant following EVLP; and (II) the contribution of that donor lung to a favorable recipient outcome. They created a model that includes donor characteristics and perfusatederived mediators of inflammation to predict, with $>70 \%$ accuracy, good transplant outcome (96). The utility of this novel EVLP diagnostic platform was further validated using prospectively collected perfusate samples (97).

\section{EVLP as a logistic tool and organ repair hub}

EVLP can safely prolong lung preservation. In fact, PGD remained low despite significantly longer preservation on EVLP $(10,98)$. The routine use of normothermic EVLP for greater than 12 hours of preservation led to excellent recipient outcomes in Toronto (10). Moreover, a prospective randomized trial by the Vienna group showed that extended preservation time on EVLP for standard criteria donor lungs did not compromise lung graft quality (11).

Total lung preservation time during EVLP is the sum of 4 distinct intervals. (I) CIT1: the period of cold static preservation from cross clamp followed by cold flush to the start of normothermic EVLP); (II) normothermic EVLP time; (III) CIT2: the period of cold static preservation after EVLP and before placement in the recipient's chest; and (IV) warm ischemic time (WIT): implantation time; the time from the placement of the graft in the chest to reperfusion $(45,99)$. Therefore, EVLP can be used to extend overall lung preservation time by splitting CIT into two short CIT periods divided by an additional EVLP time window (Figure 4A).

EVLP can have a beneficial effect on hospital logistics regarding lung transplantation; by extending total ischemic time, EVLP may help to optimize OR room availability, staffing arrangements, and transporting the transplant recipient from distant areas to the hospital (45). A recent large animal study showed that cold static preservation at $10{ }^{\circ} \mathrm{C}$ with 2 short cycles of EVLP safely extends the lung preservation period up to 3 days (100). Extended periods of EVLP will also enable complex biological treatments such as gene and cell-based therapies (Figure 4B).

Hospitals with low lung transplant volumes may not have the means or desire to integrate an EVLP program since the technology requires specialized equipment, technical experts and operating space. A centralized EVLP service 
model is a concept whereby dedicated centers are equipped for high volume EVLP assessment and reconditioning of donor lungs prior to sending to recipient lung transplant centers without an EVLP program (101). The model includes donor lung recovery and transportation to a nearby EVLP center. The donor lungs are then perfused by a trained EVLP team and offered to transplant centers in need, should the lungs satisfy acceptance criteria. Once accepted, these lungs are transported to the recipient hospital and prepared for implantation (Figure 4C) (45). An ongoing clinical trial in a facility in the US (identifier: NCT03641677) will evaluate the feasibility of this concept.

\section{Conclusions}

EVLP has emerged as an advanced preservation tool for lung transplantation, and has successfully expanded lung transplant utility by rescuing high-risk donor lungs and optimizing transplant logistics. EVLP can also serve as a critical translational research tool to bring novel therapies from bench to bedside. Several personalized treatments have been developed for use specifically during EVLP, and advanced therapies and rapid diagnostics will further help to utilize more lungs for transplantation. EVLP remains strongly positioned to continue to advance lung transplant activity worldwide.

\section{Acknowledgments}

The authors would like to thank Manyin Chen, Hemant Gokhale, and Hongchao Shan, and the team of EVLP specialists at Toronto General Hospital, University Health Network. We would like to thank Stacey Krumholtz for her excellent illustration. We would also like to thank Jerome Valero for manuscript review.

Funding: None.

\section{Footnote}

Provenance and Peer Review: This article was commissioned by the Guest Editor (Jonathan D'Cunha) for the series "Lung Transplantation: Past, Present, and Future" published in Fournal of Thoracic Disease. The article has undergone external peer review.

Conflicts of Interest: All authors have completed the ICMJE uniform disclosure form (available at https:// dx.doi.org/10.21037/jtd-2021-23). The series "Lung
Transplantation: Past, Present, and Future" was commissioned by the editorial office without any funding or sponsorship. MC is a co-founder of XOR Labs Toronto Inc., a company dedicated to the development of EVLP machines. The XOR EVLP machine was not used in the performance of this study. SK reports grants from XVIVO Perfusion, personal fees from United Therapeutics/Lung Bioengineering, during the conduct of the study; and SK is a co-founder of XOR Labs Toronto Inc., a company dedicated to the development of EVLP machines. The XOR EVLP machine was not used in the performance of this study. SK is the co-founder and Chief Scientific Officer of Perfusix Canada Inc. The authors have no other conflicts of interest to declare.

Ethical Statement: All authors are accountable for all aspects of the work in ensuring that questions related to the accuracy or integrity of any part of the work are appropriately investigated and resolved.

Open Access Statement: This is an Open Access article distributed in accordance with the Creative Commons Attribution-NonCommercial-NoDerivs 4.0 International License (CC BY-NC-ND 4.0), which permits the noncommercial replication and distribution of the article with the strict proviso that no changes or edits are made and the original work is properly cited (including links to both the formal publication through the relevant DOI and the license). See: https://creativecommons.org/licenses/by-nc-nd/4.0/.

\section{References}

1. Valapour M, Paulson K, Smith JM, et al. OPTN/SRTR 2011 Annual Data Report: lung. Am J Transplant 2013;13 Suppl 1:149-77.

2. Keeshan BC, Rossano JW, Beck N, et al. Lung transplant waitlist mortality: height as a predictor of poor outcomes. Pediatr Transplant 2015;19:294-300.

3. Tuttle-Newhall JE, Krishnan SM, Levy MF, et al. Organ donation and utilization in the United States: 1998-2007. Am J Transplant 2009;9:879-93.

4. Yeung JC, Cypel M, Waddell TK, et al. Update on donor assessment, resuscitation, and acceptance criteria, including novel techniques--non-heart-beating donor lung retrieval and ex vivo donor lung perfusion. Thorac Surg Clin 2009;19:261-74.

5. Divithotawela C, Cypel M, Martinu T, et al. Longterm Outcomes of Lung Transplant With Ex Vivo Lung 
Perfusion. JAMA Surg 2019;154:1143-50.

6. Cypel M, Yeung JC, Liu M, et al. Normothermic ex vivo lung perfusion in clinical lung transplantation. $\mathrm{N}$ Engl J Med 2011;364:1431-40.

7. Fiser SM, Tribble CG, Long SM, et al. Ischemiareperfusion injury after lung transplantation increases risk of late bronchiolitis obliterans syndrome. Ann Thorac Surg 2002;73:1041-7; discussion 1047-8.

8. Kreisel D, Krupnick AS, Puri V, et al. Short- and longterm outcomes of 1000 adult lung transplant recipients at a single center. J Thorac Cardiovasc Surg 2011;141:215-22.

9. Diamond JM, Lee JC, Kawut SM, et al. Clinical risk factors for primary graft dysfunction after lung transplantation. Am J Respir Crit Care Med 2013;187:527-34.

10. Yeung JC, Krueger T, Yasufuku K, et al. Outcomes after transplantation of lungs preserved for more than $12 \mathrm{~h}$ : a retrospective study. Lancet Respir Med 2017;5:119-24.

11. Slama A, Schillab L, Barta M, et al. Standard donor lung procurement with normothermic ex vivo lung perfusion: A prospective randomized clinical trial. J Heart Lung Transplant 2017;36:744-53.

12. Machuca TN, Cypel M, Yeung JC, et al. Protein expression profiling predicts graft performance in clinical ex vivo lung perfusion. Ann Surg 2015;261:591-7.

13. Nakajima D, Cypel M, Bonato R, et al. Ex Vivo Perfusion Treatment of Infection in Human Donor Lungs. Am J Transplant 2016;16:1229-37.

14. Nakajima D, Liu M, Ohsumi A, et al. Lung Lavage and Surfactant Replacement During Ex Vivo Lung Perfusion for Treatment of Gastric Acid Aspiration-Induced Donor Lung Injury. J Heart Lung Transplant 2017;36:577-85.

15. Machuca TN, Hsin MK, Ott HC, et al. Injury-specific ex vivo treatment of the donor lung: pulmonary thrombolysis followed by successful lung transplantation. Am J Respir Crit Care Med 2013;188:878-80.

16. Cypel M, Liu M, Rubacha M, et al. Functional repair of human donor lungs by IL-10 gene therapy. Sci Transl Med 2009; 1:4ra9.

17. Hopkinson DN, Bhabra MS, Hooper TL. Pulmonary graft preservation: a worldwide survey of current clinical practice. J Heart Lung Transplant 1998;17:525-31.

18. Fischer S, Matte-Martyn A, De Perrot M, et al. Lowpotassium dextran preservation solution improves lung function after human lung transplantation. J Thorac Cardiovasc Surg 2001;121:594-6.

19. Carrel A, Lindbergh CA. THE CULTURE OF WHOLE ORGANS. Science 1935;81:621-3.

20. Jirsch DW, Fisk RL, Couves CM. Ex vivo evaluation of stored lungs. Ann Thorac Surg 1970;10:163-8.

21. Steen S, Sjöberg T, Pierre L, et al. Transplantation of lungs from a non-heart-beating donor. Lancet 2001;357:825-9.

22. Steen S, Liao Q, Wierup PN, et al. Transplantation of lungs from non-heart-beating donors after functional assessment ex vivo. Ann Thorac Surg 2003;76:244-52; discussion 252.

23. Steen S, Ingemansson R, Eriksson L, et al. First human transplantation of a nonacceptable donor lung after reconditioning ex vivo. Ann Thorac Surg 2007;83:2191-4.

24. Ingemansson R, Eyjolfsson A, Mared L, et al. Clinical transplantation of initially rejected donor lungs after reconditioning ex vivo. Ann Thorac Surg 2009;87:255-60.

25. Erasmus ME, Fernhout MH, Elstrodt JM, et al.

Normothermic ex vivo lung perfusion of non-heartbeating donor lungs in pigs: from pretransplant function analysis towards a 6-h machine preservation. Transpl Int 2006;19:589-93.

26. Cypel M, Yeung JC, Hirayama S, et al. Technique for prolonged normothermic ex vivo lung perfusion. J Heart Lung Transplant 2008;27:1319-25.

27. Van Raemdonck D, Neyrinck A, Cypel M, et al. Ex-vivo lung perfusion. Transpl Int 2015;28:643-56.

28. Warnecke G, Van Raemdonck D, Smith MA, et al. Normothermic ex-vivo preservation with the portable Organ Care System Lung device for bilateral lung transplantation (INSPIRE): a randomised, open-label, non-inferiority, phase 3 study. Lancet Respir Med 2018;6:357-67.

29. Loor G, Warnecke G, Villavicencio MA, et al. Portable normothermic ex-vivo lung perfusion, ventilation, and functional assessment with the Organ Care System on donor lung use for transplantation from extended-criteria donors (EXPAND): a single-arm, pivotal trial. Lancet Respir Med 2019;7:975-84.

30. Aigner C, Slama A, Hötzenecker K, et al. Clinical ex vivo lung perfusion--pushing the limits. Am J Transplant 2012;12:1839-47.

31. Sage E, Mussot S, Trebbia G, et al. Lung transplantation from initially rejected donors after ex vivo lung reconditioning: the French experience. Eur J Cardiothorac Surg 2014;46:794-9.

32. Warnecke G, Moradiellos J, Tudorache I, et al. Normothermic perfusion of donor lungs for preservation and assessment with the Organ Care System Lung before bilateral transplantation: a pilot study of 12 patients. Lancet 2012;380:1851-8.

33. Zhang ZL, van Suylen V, van Zanden JE, et al. First 
experience with ex vivo lung perfusion for initially discarded donor lungs in the Netherlands: a single-centre study. Eur J Cardiothorac Surg 2019;5 5:920-6.

34. Fisher A, Andreasson A, Chrysos A, et al. An observational study of Donor Ex Vivo Lung Perfusion in UK lung transplantation: DEVELOP-UK. Health Technol Assess 2016;20:1-276.

35. Wallinder A, Ricksten SE, Silverborn M, et al. Early results in transplantation of initially rejected donor lungs after ex vivo lung perfusion: a case-control study. Eur J Cardiothorac Surg 2014;45:40-4; discussion 44-5.

36. Reeb J, Cypel M. Ex vivo lung perfusion. Clin Transplant 2016;30:183-94.

37. Galasso M, Feld JJ, Watanabe Y, et al. Inactivating hepatitis $\mathrm{C}$ virus in donor lungs using light therapies during normothermic ex vivo lung perfusion. Nat Commun 2019;10:481.

38. Machuca TN, Cypel M. Ex vivo lung perfusion. J Thorac Dis 2014;6:1054-62.

39. Yeung JC, Cypel M, Machuca TN, et al. Physiologic assessment of the ex vivo donor lung for transplantation. J Heart Lung Transplant 2012;31:1120-6.

40. Tikkanen JM, Cypel M, Machuca TN, et al. Functional outcomes and quality of life after normothermic ex vivo lung perfusion lung transplantation. J Heart Lung Transplant 2015;34:547-56.

41. Healey A, Watanabe Y, Mills C, et al. Initial lung transplantation experience with uncontrolled donation after cardiac death in North America. Am J Transplant 2020;20:1574-81.

42. Cypel M, Yeung JC, Donahoe L, et al. Normothermic ex vivo lung perfusion: Does the indication impact organ utilization and patient outcomes after transplantation? J Thorac Cardiovasc Surg 2019. [Epub ahead of print]. doi: 10.1016/j.jtcvs.2019.06.123.

43. Ceulemans LJ, Monbaliu D, Verslype C, et al. Combined liver and lung transplantation with extended normothermic lung preservation in a patient with end-stage emphysema complicated by drug-induced acute liver failure. Am J Transplant 2014;14:2412-6.

44. Loor G, Howard BT, Spratt JR, et al. Prolonged EVLP Using OCS Lung: Cellular and Acellular Perfusates. Transplantation 2017;101:2303-11.

45. Ali A, Cypel M. Ex-vivo lung perfusion and ventilation: where to from here? Curr Opin Organ Transplant 2019;24:297-304.

46. Buchko MT, Stewart CJ, Hatami S, et al. Total parenteral nutrition in ex vivo lung perfusion: Addressing metabolism improves both inflammation and oxygenation. Am J Transplant 2019;19:3390-7.

47. Takahashi M, Andrew Cheung HY, Watanabe T, et al. Strategies to prolong homeostasis of ex vivo perfused lungs. J Thorac Cardiovasc Surg 2021;161:1963-73.

48. Inci I, Zhai W, Arni S, et al. Fibrinolytic treatment improves the quality of lungs retrieved from non-heart-beating donors. J Heart Lung Transplant 2007;26:1054-60.

49. Lin H, Chen M, Tian F, et al. $\alpha 1$-Anti-trypsin improves function of porcine donor lungs during ex-vivo lung perfusion. J Heart Lung Transplant 2018;37:656-66.

50. Mariscal A, Nykanen A, Tikkanen J, et al. Alpha 1 antitrypsin treatment during human ex vivo lung perfusion improves lung function by protecting lung endothelium. J Heart Lung Transplant 2020;39:S71-2.

51. Charles EJ, Mehaffey JH, Sharma AK, et al. Lungs donated after circulatory death and prolonged warm ischemia are transplanted successfully after enhanced ex vivo lung perfusion using adenosine A2B receptor antagonism. J Thorac Cardiovasc Surg 2017;154:1811-20.

52. Frank JA, Briot R, Lee JW, et al. Physiological and biochemical markers of alveolar epithelial barrier dysfunction in perfused human lungs. Am J Physiol Lung Cell Mol Physiol 2007;293:L52-9.

53. Valenza F, Rosso L, Coppola S, et al. -adrenergic agonist infusion during extracorporeal lung perfusion: effects on glucose concentration in the perfusion fluid and on lung function. J Heart Lung Transplant 2012;31:524-30.

54. Hijiya K, Chen-Yoshikawa TF, Kondo T, et al. Bronchodilator Inhalation During Ex Vivo Lung Perfusion Improves Posttransplant Graft Function After Warm Ischemia. Ann Thorac Surg 2017;103:447-53.

55. Andreasson A, Karamanou DM, Perry JD, et al. The effect of ex vivo lung perfusion on microbial load in human donor lungs. J Heart Lung Transplant 2014;33:910-6.

56. Machuca TN, Cypel M, Bonato R, et al. Safety and Efficacy of Ex Vivo Donor Lung Adenoviral IL-10 Gene Therapy in a Large Animal Lung Transplant Survival Model. Hum Gene Ther 2017;28:757-65.

57. Lee JW, Fang X, Gupta N, et al. Allogeneic human mesenchymal stem cells for treatment of E. coli endotoxininduced acute lung injury in the ex vivo perfused human lung. Proc Natl Acad Sci U S A 2009;106:16357-62.

58. Mordant P, Nakajima D, Kalaf R, et al. Mesenchymal stem cell treatment is associated with decreased perfusate concentration of interleukin-8 during ex vivo perfusion of donor lungs after 18-hour preservation. J Heart Lung Transplant 2016;35:1245-54. 
59. Nakajima D, Watanabe Y, Ohsumi A, et al. Mesenchymal stromal cell therapy during ex vivo lung perfusion ameliorates ischemia-reperfusion injury in lung transplantation. J Heart Lung Transplant 2019;38:1214-23.

60. Nykanen A, Mariscal A, Estrada C, et al. Engineered mesenchymal stromal cell therapy during human lung ex vivo lung perfusion is affected by lung acidosis. J Heart Lung Transplant 2020;39:S178.

61. Lonati C, Bassani GA, Brambilla D, et al. Mesenchymal stem cell-derived extracellular vesicles improve the molecular phenotype of isolated rat lungs during ischemia/reperfusion injury. J Heart Lung Transplant 2019;38:1306-16.

62. Miyamoto E, Martinu T, Hwang D, et al. Intrapulmonary immune regulation by pre-transplant infusion of recipientderived regulatory t cells in a rat model of ex vivo lung perfusion followed by transplantation. J Heart Lung Transplant 2019;38:S40-1.

63. Cypel M, Feld JJ, Galasso M, et al. Prevention of viral transmission during lung transplantation with hepatitis C-viraemic donors: an open-label, single-centre, pilot trial. Lancet Respir Med 2020;8:192-201.

64. Wallinder A, Hansson C, Dellgren G. Hemoconcentration in ex vivo lung perfusion: a case report of a novel technique used in clinical lung transplantation. J Thorac Cardiovasc Surg 2013;145:e76-7.

65. Nilsson T, Hansson C, Wallinder A, et al. Hemofiltration in ex vivo lung perfusion-a study in experimentally induced pulmonary edema. J Thorac Cardiovasc Surg 2016;151:570-5.e1.

66. Iskender I, Cosgun T, Arni S, et al. Cytokine filtration modulates pulmonary metabolism and edema formation during ex vivo lung perfusion. J Heart Lung Transplant 2017. [Epub ahead of print]. doi: 10.1016/ j.healun.2017.05.021.

67. Niikawa H, Okamoto T, Ayyat KS, et al. The protective effect of prone lung position on ischemia-reperfusion injury and lung function in an ex vivo porcine lung model. J Thorac Cardiovasc Surg 2019;157:425-33.

68. Oto T, Excell L, Griffiths AP, et al. The implications of pulmonary embolism in a multiorgan donor for subsequent pulmonary, renal, and cardiac transplantation. J Heart Lung Transplant 2008;27:78-85.

69. Inci I, Yamada Y, Hillinger S, et al. Successful lung transplantation after donor lung reconditioning with urokinase in ex vivo lung perfusion system. Ann Thorac Surg 2014;98:1837-8.

70. Luc JG, Bozso SJ, Freed DH, et al. Successful repair of donation after circulatory death lungs with large pulmonary embolus using the lung organ care system for ex vivo thrombolysis and subsequent clinical transplantation. Transplantation 2015;99:e1-2.

71. Meers C, Van Raemdonck D, Verleden GM, et al. The number of lung transplants can be safely doubled using extended criteria donors; a single-center review. Transpl Int 2010;23:628-35.

72. Diamond JM, Arcasoy S, Kennedy CC, et al. Report of the International Society for Heart and Lung Transplantation Working Group on Primary Lung Graft Dysfunction, part II: Epidemiology, risk factors, and outcomes-A 2016 Consensus Group statement of the International Society for Heart and Lung Transplantation. J Heart Lung Transplant 2017;36:1104-13.

73. Inci I, Ampollini L, Arni S, et al. Ex vivo reconditioning of marginal donor lungs injured by acid aspiration. J Heart Lung Transplant 2008;27:1229-36.

74. Inci I, Hillinger S, Arni S, et al. Reconditioning of an injured lung graft with intrabronchial surfactant instillation in an ex vivo lung perfusion system followed by transplantation. J Surg Res 2013;184:1143-9.

75. Guimarães S, Moura D. Vascular adrenoceptors: an update. Pharmacol Rev 2001;53:319-56.

76. Ruiz I, Gavaldà J, Monforte V, et al. Donor-to-host transmission of bacterial and fungal infections in lung transplantation. Am J Transplant 2006;6:178-82.

77. Nykanen A, Mariscal A, Takahashi M, et al. Engineered mesenchymal stromal cell therapy during pig ex vivo lung perfusion and transplant. J Heart Lung Transplant 2019;38:S13-4.

78. Koike T, Yeung JC, Cypel M, et al. Kinetics of lactate metabolism during acellular normothermic ex vivo lung perfusion. J Heart Lung Transplant 2011;30:1312-9.

79. Aboelnazar NS, Himmat S, Hatami S, et al. Negative pressure ventilation decreases inflammation and lung edema during normothermic ex-vivo lung perfusion. J Heart Lung Transplant 2018;37:520-30.

80. Buchko MT, Himmat S, Stewart CJ, et al. Continuous Hemodialysis Does Not Improve Graft Function During Ex Vivo Lung Perfusion Over 24 Hours. Transplant Proc 2019;51:2022-8.

81. de Perrot M, Liu M, Waddell TK, et al. Ischemiareperfusion-induced lung injury. Am J Respir Crit Care Med 2003;167:490-511.

82. Kakishita T, Oto T, Hori S, et al. Suppression of inflammatory cytokines during ex vivo lung perfusion with an adsorbent membrane. Ann Thorac Surg 2010;89:1773-9.

83. Cornejo RA, Díaz JC, Tobar EA, et al. Effects of prone 
positioning on lung protection in patients with acute respiratory distress syndrome. Am J Respir Crit Care Med 2013;188:440-8.

84. Watanabe Y, Galasso M, Watanabe T, et al. Donor prone positioning protects lungs from injury during warm ischemia. Am J Transplant 2019;19:2746-55.

85. Iskender I, Sakamoto J, Nakajima D, et al. Human $\alpha 1$ antitrypsin improves early post-transplant lung function: Pre-clinical studies in a pig lung transplant model. J Heart Lung Transplant 2016;35:913-21.

86. Gao W, Zhao J, Kim H, et al. $\alpha 1$-Antitrypsin inhibits ischemia reperfusion-induced lung injury by reducing inflammatory response and cell death. J Heart Lung Transplant 2014;33:309-15.

87. Mariscal A, Caldarone L, Chen M, et al. Alpha 1 antitrypsin to prevent ischemia repercusion injury in a pig lung transplant survival model. J Heart Lung Transplant 2018;37:S81.

88. Fischer S, Liu M, MacLean AA, et al. In vivo transtracheal adenovirus-mediated transfer of human interleukin-10 gene to donor lungs ameliorates ischemia-reperfusion injury and improves early posttransplant graft function in the rat. Hum Gene Ther 2001;12:1513-26.

89. de Perrot M, Fischer S, Liu M, et al. Impact of human interleukin-10 on vector-induced inflammation and early graft function in rat lung transplantation. Am J Respir Cell Mol Biol 2003;28:616-25.

90. Yeung JC, Wagnetz D, Cypel M, et al. Ex vivo adenoviral vector gene delivery results in decreased vector-associated inflammation pre- and post-lung transplantation in the pig. Mol Ther 2012;20:1204-11.

91. Machuca TN, Cypel M, Zhao Y, et al. The role of the endothelin-1 pathway as a biomarker for donor lung assessment in clinical ex vivo lung perfusion. J Heart Lung Transplant 2015;34:849-57.

92. Andreasson ASI, Borthwick LA, Gillespie C, et al. The role of interleukin- $1 \beta$ as a predictive biomarker and potential therapeutic target during clinical ex vivo lung

Cite this article as: Watanabe T, Cypel M, Keshavjee S. Ex vivo lung perfusion. J Thorac Dis 2021;13(11):6602-6617. doi: $10.21037 /$ jtd-2021-23 perfusion. J Heart Lung Transplant 2017;36:985-95.

93. Hashimoto K, Cypel M, Juvet S, et al. Higher M30 and high mobility group box 1 protein levels in ex vivo lung perfusate are associated with primary graft dysfunction after human lung transplantation. J Heart Lung Transplant 2017. [Epub ahead of print]. doi: 10.1016/ j.healun.2017.06.005.

94. Mallavia B, Liu F, Lefrançais E, et al. Mitochondrial DNA Stimulates TLR9-Dependent Neutrophil Extracellular Trap Formation in Primary Graft Dysfunction. Am J Respir Cell Mol Biol 2020;62:364-72.

95. Caldarone L, Mariscal A, Sage A, et al. Neutrophil extracellular traps in ex vivo lung perfusion perfusate predict the clinical outcome of lung transplant recipients. Eur Respir J 2019;53:1801736.

96. Sage A, Richard-Greenblatt M, Zhong K, et al. Validation of an evlp perfusate diagnostic test for the prediction of lung transplant outcomes. J Heart Lung Transplant 2019;38:S53.

97. Chao BT, Sage AT, Bai X, et al. Validation of a rapid molecular assessment platform and cytokine score for integration with ex vivo lung perfusion (evlp) assessment. J Heart Lung Transplant 2020;39:S112.

98. Cypel M, Yeung JC, Machuca T, et al. Experience with the first 50 ex vivo lung perfusions in clinical transplantation. J Thorac Cardiovasc Surg 2012;144:1200-6.

99. Cypel M, Yeung JC, Keshavjee S. Introducing the concept of semielective lung transplantation through the use of ex vivo lung perfusion. J Thorac Cardiovasc Surg 2018;156:2350-2.

100.Ali A, Nykanen A, Brambate E, et al. Successful transplantation of porcine lungs following 3 days of preservation using a modified cold static method paired with intermittent normothermic ex vivo lung perfusion (evlp). J Heart Lung Transplant 2020;39:S353.

101. Yeung JC, Cypel M, Keshavjee S. Ex-vivo lung perfusion: the model for the organ reconditioning hub. Curr Opin Organ Transplant 2017;22:287-9. 